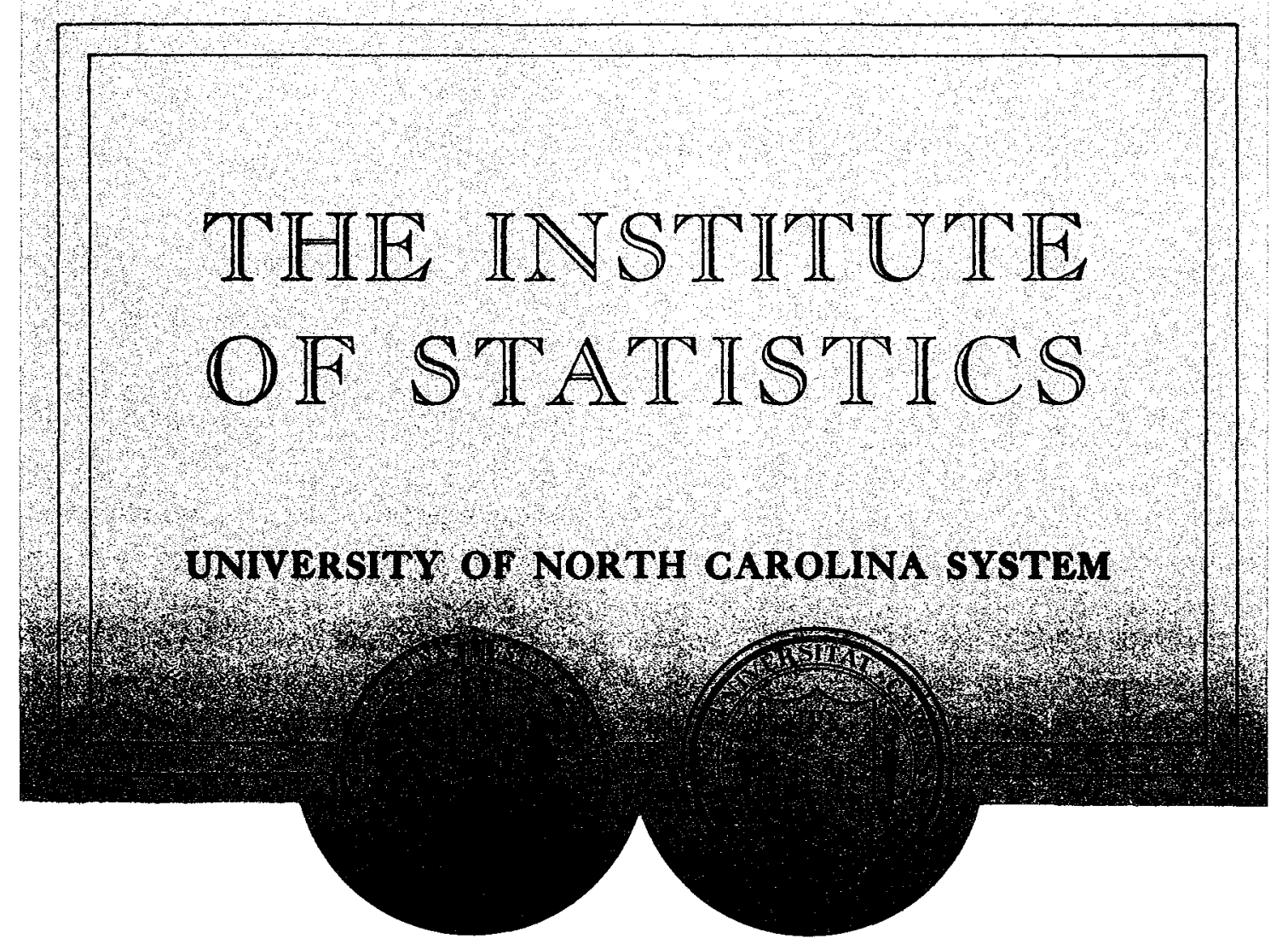

Effects of afterhyperpolarization on neuronal firing

P. Lansky, M. Musila and C. E. Smith

Institute of Statistics Mimeograph Series No. 2204

September 1991

NORTH CAROLINA STATE UNIVERSITY

Raleigh, North Carolina 


\title{
Effects of afterhyperpolarization on neuronal firing
}

\author{
P. Lansky, M. Musila and C. E. Smith \\ Institute of Statistics Mimeograph Series No. 2204
}

September 1991 


\section{Effects of afterhyperpolarization on neuronal firing}

P. Lánsky ${ }^{1}$, M. Musila ${ }^{2}$ and C.E. Smith ${ }^{3}$

${ }^{1}$ Institute of Physiology, Czechoslovak Academy of Sciences, Prague, Czechoslovakia

${ }^{2}$ Institute of Biophysics, 3rd Medical School, Charles University, Prague, Czechoslovakia

${ }^{3}$ Department of Statistics, North Carolina State University, Raleigh, NC 27695, USA

Address for correspondence:

Dr. Petr Lánský,

Institute of Physiology,

Czechoslovak Academy of Sciences,

Vídeñská 1083,

CS-142 20 Prague 4,

Czechoslovakia 
Abstract. Stein's model for a neuron is studied. This model is modified to take into account the effects of afterhyperpolarization on the neuronal firing. The relative refractory phase, following the absolute one, is modelled by a time-increasing amplitude of postsynaptic potentials and it is also incorporated into the model. Besides the simulation of the model, some theoretical results and approximation methods are derived. Afterhyperpolarization tends to preserve the linearity of the frequency transfer characteristic and it has a limited effect on the moments of the interspike intervals in general. The main effects are seen at high firing rates and in the removal of short intervals in the interspike interval histogram.

\section{Introduction}

Stochastic models of single neuron activity have recently been reviewed by Tuckwell $(1988,1989)$. In most of these models the membrane depolarization from the resting potential is described by a onedimensional stochastic process $X=\{X(t), t \geq 0\}$. The process $X$ is called the membrane potential. The random synaptic inputs evoke changes in the trajectory of $\mathrm{X}$. The model neuron produces an action potential when $\mathrm{X}$ exceeds a threshold level $\mathrm{S}$ for the first time. After each firing, $\mathrm{X}$ is reset to the initial depolarization $\mathrm{X}(0)$ and subsequent inputs lead to another discharge. The resting potential is usually transformed to zero and the initial depolarization is also often assumed to be equal to the resting level, i.e. $\mathrm{X}(0)=0$. The neuronal firing corresponds to the first passage time (FPT) for the associatedstochastic process $\mathrm{X}$. The theoretical counterpart of the interspike interval (ISI) is a random variable $\Gamma^{*}$ given by the relationship

$$
\mathrm{T}^{*}=\inf \{\mathrm{t} \geq 0 ; \mathrm{X}(\mathrm{t}) \geq \mathrm{S}, \mathrm{X}(0)=0\}
$$

'Lhe neurophysiological basis of the model can be improved by incorporating an absolute refractory phase following each firing, during which the cell is inexcitable for a period $T_{\mathbf{R}}$. Then the ISI is the random variable $T$ defined as

$$
\mathrm{T}=\mathrm{T}^{*}+\mathrm{T}_{\mathrm{R}}
$$


Analytical solutions for the FPT problem are available only for the simplest neuronal models. Therefore the description of the models is often restricted to the moments of the FPT. Primarily the mean $E(T)$, the variance $V(T)$ and the coefficient of variation $C V$, defined as the ratio between the standard deviation and the mean, are computed. It is usually assumed that coding of information in the nervous system is by the firing frequency $f$ of the neuron, which is usually defined as the reciprocal value of T. The average firing frequency is often computed as the reciprocal of the mean ISI, rather then the mean of the reciprocal of the ISI, (Walloe, 1970; Tuckwell, 1978; Levine 1982; Wilbur and Rinzel, 1983). To get a better insight into the time dynamics of the neuronal firing the higher moments, skewness $\beta_{1}$ and kurtosis $\beta_{2}$, of the ISI are also sometimes computed from the experimental recordings; Correia and Landolt (1977), Lánský and Radil (1987). There have been attempts to use them for approximation of the distribution of ISI's.

The models in which the activity is described only as the FPT, are usually of the renewal character, i.e. the ISI's are independent and identically distributed random variables. This fact has been tested on experimental data from different types of neurons mainly by computing the serial correlation coefficients (e.g., Correia and Landolt, 1977; Floyd et al., 1982; Yamamoto and Nakahama, 1983; Lánský and Radil, 1987; Surmeier et al., 1989) and with different results. It means that for the activity of a given type of neuron the serial correlations reveal that the previous ISI's may influence the following ISI, however, this is not always a systematical phenomenon. The dependency in the ISI's can be related in several ways to the state of the neuron after an action potential, also the synaptical input may be correlated and thus to cause the neuron's output to be correlated as well.

Most papers on the modelling of single neuron activity have dealt with the relationship between the random synaptic bombardment and the membrane potential behaviour. Relatively less effort has been devoted to other phenomena, which may also influence the state of the cell and modify the neuronal discharge. Some examples are: refractory behavior (Clay and Goel, 1973), recurrent 
inhibition (Tuckwell, 1978), net oscillations (Wilbur and Rinzel, 1983), afterdepolarization (Tipans and Lavendels, 1983), spatial facilitation (Lánský and Musila, 1991).

The aim of this study is to examine the role of afterhyperpolarization (AHP) on neuronal firing. An AHP has been incorporated in some models already. The model of Geisler and Goldberg (1066) had a time-varying threshold and the initial depolarization depended on the length of the previons ISI. Tuckwell (1978) used a decaying threshold for this purpose, Smith and Goldberg (1986) applied a time-varying potassium conductance in their type of neuronal model of cumulative AHP. Randomly distributed initial depolarization (Lánský and Smith, 1989; Lánský and Musila, 1991) can be also interpreted as due to an AHP. Here we attempt, on the basis of experimental data, to model the trajectory of the afterhyperpolarizing potential and to investigate how an AIP can affect some the statistical properties of ISl's.

\section{Quantitative description of AIIP}

The time course of an action potential can be divided into three phases - overshoot, afterdepolarization and afterhyperpolarization (see e.g. Ganong, 1985). The duration of the action potential wave from its onset to the first crossing of the resting level is called the absolute refractory period $T_{R}$. Due to its additive role with respect to ISI as it is defined by (1.2), we may assume for notational simplicity that $\mathrm{T}_{\mathrm{R}}=0$ here. The duration and amplitude of these phases have been measured many times in a varicty of neurons (see e.g. Grantyn and Grantyn, 1978 for references). The AHP is commonly characterized by: the time to maximum $\left(\mathrm{T}_{\mathrm{H}}\right)$, the maximum amplitude $(\mathrm{H})$ and the total duration $\left(\mathrm{T}_{\mathrm{E}}\right.$ ) (Fig. 1). The total duration is defined as the time after which the membrane potential returns sufficiently close to the resting level (Brock et al., 1952). $\mathrm{T}_{\mathrm{E}}$ defined in this way is a vague term. A more quantitative definition is given by Weems and Szsurszewski (1978). They measured $T_{E}$ as the time when the voltage has returned to $10 \%$ of its maximum value, H. In some neurons the AHP is particularly pronounced and the present model is intended for such cells. 
On the basis of numerous observations on cat spinal motoneurons Eccles (1957) concluded that the size, but not the time course, of the AHP changed greatly when the cell was electrically stimulated. While the total duration $T_{E}$ and time to the maximum $T_{H}$ seem to be fixed for a particular type of neuron, the amplitude $H$ varies for each action potential (see the comparison of several experimental records presented in Fig. 28 of the cited monograph). For the determination of $\mathrm{H}$ we turn to the experimental results of Coombs et al., (1955). These authors devised an ingenious technique of adjusting the membrane potential to any desired level by introducing a double-barreled microclectrode into the cell. They were thus able to record the action potentials evoked by a brief current pulse when the membrane potential was at various levels prior to current injection. The results were obtained from an extensive series of records and the authors stated: "The amplitude of the after potential had an approximately linear relationship to the membrane potential at which it occurred." Their results are shown in Fig. 11, 12 in the cited paper and also in several monographs (Eccles, 1957; Ruch et al., 1968).

From the shape of numerous experimental records of AHP (Brock et al., 1952; Coombs et al., 1955) it seems appropriate to model the trajectory of the AHP by the formula

$$
x(t)=-A t^{b} \exp (-t / \theta)
$$

where $A, b$ and $\theta$ are constants. Note this is similar in form to a Gamma probability density. The constant $\theta$ is determined by the total duration $T_{E}$, which is known from experiments. The other two parameters have to be determined on the basis of two other experimentally available characteristics; namely the time to maximum $\mathrm{T}_{\mathrm{H}}$ and the maximum $\mathrm{H}$. Using these values, we can establish that

$$
\mathrm{b}=\mathrm{T}_{\mathrm{H}} / \theta
$$

and for $A$ we can derive the expression 


$$
A=-H \exp \left\{\frac{\mathrm{T}_{\mathrm{H}}\left(1-\ln \left(\mathrm{T}_{\mathrm{H}}\right)\right)}{\theta}\right\}
$$

However as noted above, $H$ changes with each action potential. We define the final level, denoted by $\mathrm{X}_{\mathrm{F}}$, as the level of the membrane potential that preceded the brief current stimulus, which produce the threshold crossing. Then the size of the AIP amplitude $H$ is given by the linear dependency

$$
\mathrm{H}=\mathrm{kX} \mathrm{X}_{\mathrm{F}}+\mathrm{q}
$$

Substituting (2.2), (2.3) and (2.4) into (2.1), the time course of the AHP in the following ISI is given by the function

$$
x(t)=-\left(k X_{F}+q\right)\left(\frac{t}{T_{H}}\right)^{T_{H} / \theta} \exp \left(\left(T_{H}-t\right) / \theta\right)
$$

The resting potential of the observed cat spinal motoneurons (Coombs et al., 1955) varied from -76 to $-79 \mathrm{mV}$. If we take the mean, $-77.5 \mathrm{mV}$, as the resting potential and reference $\mathrm{X}_{\mathrm{F}}$ to it, then the values of parameters in (2.5) are approximately $k=0.375$ and $q=4.6875$ for the experimental datia in question.

\section{SLein's model involving AIIP}

Stein (1965) introduced a stochastic model of single neuron activity which incorporates several physiological features of real neurons. The model and its modifications have been used as a basis for many studies devoted to the theoretical description of neuronal activity (e.g., Tuckwell, 1979; Wilbur and Rinzel, 1983; Smith and Smith, 1984; Musila and Lánský, 1991). For simplicity we will use Stein's model with excitation only, as is commonly done for neurons with negligible inhibition (Tsurui and Osaki, 1976; Vasudevan and Vittal, 1982; Wilbur and Rinzel, 1982). The concept of the model is as 
follows: synaptic activation of a neuron leads to an excitatory postsynaptic potential (EPSP), which is characterized by a short rise time. The corresponding change in the membrane potential is modelled by a step discontinuity. The stream of EPSP's is assumed to be Poisson. In the absence of excitation the membrane potential decays exponentially towards its resting value. As a result, the behaviour of the membrane potential $\mathrm{X}$ can be described by the stochastic differential equation

$$
\mathrm{dX}=-\frac{1}{\tau} \mathrm{Xdt}+\operatorname{adP}(\mathrm{t}), \quad \mathrm{X}(0)=0
$$

where $\tau>0, \mathrm{a}>0$ are constants, $\mathrm{P}(\mathrm{t})$ is a homogeneous Poisson process with $\mathrm{P}(0)=0$ and intensity $\lambda$. The value a represents the EPSP amplitude and $\tau$ is the membrane time constant. For a neurophysiological justification of this model see Tuckwell $(1976 a, 1978)$.

The starting point of $X$ is taken to be $t=0$ and the absolute refractory period $T_{R}$ is in accordance with (1.2) simply added to FPT of the above model. The relative refractory phase, following the absolute one, has often been modelled by a time-decaying threshold (e.g., Vasudevan and Vittal, 1982; Tuckwell and Wan, 1984). There the value of the threshold is high following the spike and it decays in time to some asymptotic level. We propose another method to describe the reduced excitability of the cell immediately following the spike. For example, Katayama (1971) found that the strength of stimulus required to evoke a second impulse decreased exponentially with increase of the interval between the first and the second spike. We take the time course of the EPSP's to be

$$
a(t)=a(1-\exp (-t / \kappa))
$$

where $\kappa>0$ is a constant. Substituting (3.2) into (3.1) the model takes the form

$$
d X=-\frac{1}{\tau} X d t+a(1-\exp (-t / \kappa)) d P(t), \quad X(0)=0
$$

'This method tries to model the initial decrease in BISP amplitude due to the increased conductance of 
the neuron following the spike. This process usually takes only a few milliseconds (Schmidt, 1984).

From the equation (3.3) it follows that each membrane potential trajectory starts from the resting value and remains there until the first EPSP. We need to incorporate the AHP into this model. As the experimental technique of Coombs et al., (1955) did not significantly alter the ion conductances (Ruch et al., 1968), then we can assume that the dependency (2.4) also holds between the amplitude H of AHP and the membrane potential level at the time of the final EPSP, which caused the previous neuronal firing. In this way we are able to determine the value of II. The parameters of the AHP function (2.1) are now determined, i.e. the initial trajectory of the membrane potential can be calculated. Note that since $\mathrm{X}_{\mathrm{F}}$ is a random variable, then $\mathrm{H}$ is also. This is supported by several experimental findings where the mean and standard deviation of $H$ are presented (Grantyn and Grantyn, 1978; O'Neill et al., 1986). As follows from (2.4), for the model (3.1), the values of $H$ fall within the interval $\left(\mathrm{II}_{\min }, \mathrm{II}_{\max }\right)$, where

$$
\begin{gathered}
\mathrm{H}_{\min }=\mathrm{k}(\mathrm{S}-\mathrm{a})+\mathrm{q} \\
\mathrm{I}_{\max }=\mathrm{kS}+\mathrm{q}
\end{gathered}
$$

The tolal duration of the AHP may often be longer than $100 \mathrm{~ms}$ (Eccles et al., 1958, Weems and Szsurszewski, 1978). During this time the cell is likely to be synaptically activated. We assume the following rules of the membrane potential trajectory:

1. At time $t=0$ the AHP given by the equation (2.5) starts. The parameters $T_{H}, \theta$ (corresponds $T_{E}$ ) are constants and $H$ is computed from $X_{F}$ which is known from the previous ISI. The absolute refractory period $T_{R}$ can simply be added to the FPT.

2. Synaptic activation (EPSP) at time $t_{1}>O$ (generated in accordance with the homogeneous Poisson process appearing in (3.3)) causes the membrane potential to increase instantaneously by the amount $a\left(t_{1}\right)$ given by (3.2). Provided the membrane potential is still below the resting level, the trajectory 
continues for $t>t_{1}$ in accordance with (2.5) with parameters $T_{H I}, \theta$. However, the value of $A$ is reset at time $t_{1}$. The new value, $A_{1}$, of $A$ is determined from (2.1) in such a way that

$$
-A_{1} t_{1}^{b} \exp \left(-t_{1} / \theta\right)=x\left(t_{1}\right)+a\left(t_{1}\right)
$$

This algorithm for the evaluation of the membrane potential continues until the membrane potential crosses the resting level.

3. $\Lambda$ s soon as the membrane potential exceeds the resting level for the first time, the trajectory is determined solely by equation (3.3), that is, the behaviour of $\mathrm{X}$ starts at a random initial value from the interval $(0, S)$ at a random time determined by the rule 2 . above.

The biological basis for the rule 2. is that the neural recovery processes, which are responsible for AIP, may continue even if EPSP's are present (Ganong, 1985). Also Weems and Szurszewski (1978) found that subthreshold synaptic inputs to a neuron shortly after a spike did not appear to alter the basic properties of the AHP process.

The trajectory of the membrane potential described by these rules is illustrated in Fig. 2. We can see that an EPSP arrives before the time $\mathrm{T}_{\mathrm{H}}$, then the actual minimal value of the AHP wave; denoted by $X_{M}$, is smaller than the preasigned value $H$ computed from (2.4). The definition of $H$ and $X_{M}$ is illustrated in Fig. 2.

\section{Compuler simulations and results}

Tuckwell (1976b) derived a system of differential-difference equations for determining the FPT moments in Stein's model (3.1). However, by incorporating the relative refractory phase and AHP into the model an analytical solution becomes difficult to obtain. Therefore we performed computer simulations of the FP'T"s of the model neuron characterized in the previous section. The program language 'TUR.BO PASCAL 5.5 and an IBM compatible PC were used. 
The parameters of the function (2.1) describing the AHP trajectory were chosen according to the average values of $T_{H}$ and $T_{E}$ found experimentally by Brock et al., (1952) for cat spinal motoneurons, i.e., $\mathrm{T}_{\mathrm{H}}=14 \mathrm{~ms}$ and $\theta=20 \mathrm{~ms}$ (which corresponds to $\mathrm{T}_{\mathrm{E}} \approx 100 \mathrm{~ms}$ ). The parameters of the linear function (2.4) were taken to be $k=0.375$ and $q=4.6875$ as suggested by the experimental results of Coombs et al., (1955) on cat spinal motoneurons. Since the value of H depends via (2.4) on the preceding final level $\mathrm{X}_{\mathrm{F}}$, the initial value of $\mathrm{H}$ at the beginning of each simulation was chosen as that corresponding to the mean of the range of $X_{F}$, that is, $S-a / 2$. This choice could not have any effect on the results obtained because each simulation contains 5000 ISI's. The absolute refractory period, $T_{R}$, was always set to $1.5 \mathrm{~ms}$. The parameter, $\kappa$, of the relative refractory phase modelled by (3.2) has a value of $1 \mathrm{~ms}$ in accordance with the characterization of this phenomena in Schmidt (1984). Note that the time course of the relative refractory phase is much faster than that of the AIIP.

For the first set of simulations, the neurophysiological parameters obtained from cat spinal motoneurons and employed in Tuckwell (1978) were used: $\mathrm{S}=12 \mathrm{mV}, \tau=5.8 \mathrm{~ms}$ and $\mathrm{a}=3.2 \mathrm{mV}$. There were three aims in this first set of simulations. The first was to show the difference between the original Stein's model (3.1) and its modification with the relative refractory phase given by (3.3). The dependence of the mean output frequency $f$ on the frequency of cell excitation $\lambda$ is plotted in Fig. $3 a$. The curve $(\alpha)$ and $(\beta)$ corresponds to the model (3.1) and (3.3), respectively. As expected, with an incrensing frequency of excitation the values of $\mathrm{f}$ begin to differ due to the time-dependency of EPSP sizes in the model (3.3). This is not a large effect, as can be documented by the following results: for a high activation level, $\lambda=1000 \mathrm{~s}^{-1}$, the statistical characteristics of the ISI's were $\mathrm{E}(\mathrm{T})=7.25 \mathrm{~ms}, \mathrm{CV}$ $=0.46$ for the model $(3.1)$ and $\mathrm{E}(\mathrm{T})=8.32 \mathrm{~ms}, \mathrm{CV}=0.40$ in the other case.

The second aim was to investigate the influence of the AHP on neuronal firing in the model (3.3). From the comparison of the curves $(\beta)$ and $(\gamma)$ in Fig. 3a., with the latter obtained from the model (3.3) including an $\Lambda$ IIP, it follows that the $\Lambda$ IIP did not have a substantial effect on the shape 
of the Irequency transfer characteristic. The corresponding values of $f$ did not differ by more than $12 \%$ (compared with model (3.3)). Also the variability of the ISI's was not greatly changed by the introdiction of an AHP. For example with $\lambda=800 \mathrm{~s}^{-1}$, the moments of the ISI's equaled $\mathrm{E}(\mathrm{T})=$ $10.50 \mathrm{~ms}, \mathrm{CV}=0.47$ in the model $(3.3)$ and $\mathrm{E}(\mathrm{T})=11.92 \mathrm{~ms}, \mathrm{CV}=0.44$ for its modification involving an AHP.

We were interested not only in the changes of the frequency transfer characteristics but also how the introduced modifications of the model (3.1) changed the variability of the firing patterns. The changes of the coefficient of variation are illustrated in Fig. 3b. It can be seen that the model (3.3) is characterized by a lower variability with respect to (3.1) and the effect of the AHP suppressed the variability even more. The differences remained almost constant over the range of studied output frequencies $\lambda$. The changes of the higher moments (skewness $\beta_{1}$ and kurtosis $\beta_{2}$ ) are documented in Fig. 3c. There is an apparent decrease of $\beta_{2}$ with increasing input excitation rate $\lambda$ while the decrease of $\beta_{1}$ is much smaller. Generally, these coefficients took the values in rather narrow range $\left(\beta_{1} \in\right.$ $\left.(1.39,1.90), \quad \beta_{2} \in(3.37,5.12)\right)$ when compared with those obtained experimentally (Correia and Landolt, 1977; Lánský and Radil, 1987).

The third objective of the first set of simulations was to characterize the amplitude of the AHP as a random variable. The distribution of the maximum amplitude $H$ of the AHP, as computed from the previous trajectory's $\mathrm{X}_{\mathrm{F}}$, in the simulation for $\lambda=200 \mathrm{~s}^{-1}$ is shown in Fig. 4a. Here the moments of $\mathrm{Il}$ were as follows: a mean of $8.31 \mathrm{mV}$, a standard deviation of $0.255 \mathrm{mV}$ and their range was $\mathrm{H}_{\min }$ $=7.99 \mathrm{mV}, \mathrm{H}_{\max }=9.19 \mathrm{mV}$. The histograms of minimal level of the membrane potential trajectory $X_{M}$ during the $A H P$ are shown in Fig. $4 b$ and of course they are smaller and with a tendency to decrease with increasing $\lambda$. One may expect that for low values of $\lambda$ the distributions of $H$ and $X_{M}$ are similar. This is illustrated in Fig. 4c., where two first moments of $\mathrm{X}_{M}$ are plotted against $\lambda$. The average of $X_{M}$ exponentially increases with decreasing input frequency suggesting a tenclency to attain a value in the range of II. The standard deviation of $x_{M}$ has a linear trend but not to a value 
corresponding to the range of $H$. It means that for the very low input frequencies $\lambda$ there must appear a new qualitative development of the standard deviation of $\mathrm{X}_{\mathrm{M}}$.

The output characteristics of the model in this first set of simulations were compared in the case of the variable amplitude of the AHP and the constant amplitude fixed at its minimal value, $\mathrm{H}_{\min }$ , $=7.99 \mathrm{mV}$. The variability of the amplitude of AHP has no effect on the firing patterns for the considered parameters. This result would permit to assign the same AHP form to the diffusion type models derived from Stein's model, Lánský (1984)

There exists a great variability in the EPSP sizes (Lev-Tov et al., 1983; MacDonald et al., 1983; Neale et al., 1983; Nelson et al., 1983). In some neurons the EPSP amplitudes may be even greater than the distance between the resting potential and the threshold. Such neurons have been usually modelled by the gating or the selective interaction models (e.g., Smith and Smith, 1965; Coleman and Gastwirth, 1970; Lánský et al., 1981). If we model such a neuron by equation (3.3), after the time

$$
\mathrm{T}_{\mathrm{D}}=-\kappa \ln \left(1-\frac{\mathrm{S}}{\mathrm{a}}\right)
$$

each EPSP leads to a threshold crossing. However, if we augment this model by incorporating an AHP, then the action potential production can be rather different. This situation was studied using the parameters: $\mathrm{S}=5 \mathrm{mV}$ (Coombs et al., 1959), $a=6 \mathrm{mV}, \tau=50 \mathrm{~ms}$ (Eccles, 1957). Let us realize that for this choice of $S$ and a the value of $\tau$ does not play any role. The refractoriness and AHP parameters remained unchanged from that used in the first set of simulations. For comparison we computed the mean output frequency $f$ as a function of input frequency $\lambda$ for the model (3.3) and its modification involving an AHP. The percentage difference between corresponding values of the frequency transfer characteristics is plotted in Fig. 5. From the figure it clearly follows that the influence of the AIIP on neuronal firing is considerable for the input frequency range $30-80 \mathrm{~s}^{-1}$ where 
the differences exceed $40 \%$. For lower or higher frequencies of cell excitation the differences become smaller. 'The effect of an AIIP on the shape of ISI distribution is shown in the case of $\lambda=50 \mathrm{~s}^{-1}$. Here the mean output frequencies differ by approximately $43 \%$. The ISI histograms for both models are illustrated in Fig. 6. As expected, the AHP causes a more gradual initial slope, a broader peak and in general longer ISI's. 'The statistical characteristics are $\mathrm{E}(\mathrm{T})=23.53 \mathrm{~ms}, \mathrm{CV}=0.88$ and the maximum ISI is $188,58 \mathrm{~ms}$ for the model $(3.3)$, while $\mathrm{E}(\mathrm{T})=40.82 \mathrm{~ms}, \mathrm{CV}=0.65$ and the maximum ISI is 204.03 $\mathrm{ms}$ for its modification involving an AHP. If $\kappa \rightarrow \infty$ in the model (3.3), i.e., no relative refractoriness, and there is no AHP, the ISI distribution will be an exponential probability density function with $\mathrm{E}(\mathrm{T})=20 \mathrm{~ms}$ and $\mathrm{CV}=1.00$.

The first five serial correlation coefficients were estimated in a standard way (e.g. Tuckwell, 1988). The obtained serial correlograms were tested at $5 \%$ significance level with only occasional serial correlation ontside the acceptable limit for independence. The serial correlation at lag 1 in no case permitted to reject the null hypothesis of independence of neighboring ISI's.

\section{5 'Thcoretical results}

For model (3.3) withont an $\Lambda \mathrm{HP}$, the mean voltage trajectory, $\mathrm{E}[\mathrm{X}]$, for $\tau \neq \kappa$, is given by

$$
\mathrm{E}[\mathrm{X}(\mathrm{t})]=\lambda \mathrm{a} \tau+\left(-\lambda \mathrm{a} \tau+\frac{\lambda \mathrm{a} \tau \kappa}{\kappa-\tau}\right) \mathrm{e}^{-\mathrm{t} / \tau}-\frac{\lambda \mathrm{a} \tau \kappa}{\kappa-\tau} \mathrm{e}^{-\mathrm{t} / \kappa}
$$

and for $\tau=\kappa$,

$$
\mathrm{E}[\mathrm{X}(\mathrm{t})]=\lambda \mathrm{a} \tau+(-\lambda \mathrm{a} \tau-\lambda \mathrm{at}) \mathrm{e}^{-\mathrm{t} / \tau}
$$

where $\lambda a \tau$ is the mean asymptotic level for the trajectory and is the same level as that for the model withont any relative refractoriness. For the parameters used for the first set of simulations, $\tau=5.8$ ms, $\mathrm{T}_{\mathrm{R}}=1.5 \mathrm{~ms}$ and $\kappa=1 \mathrm{~ms}$, the terms in (5.1) are arranged in decreasing order of their rate of decay. 'The last term prevents an analytic solution for the time $t^{*}$ at which the mean trajectory crosses 
the threshold, $S=12 \mathrm{mV}$. If we neglect this term and approximate $t^{*}$, we can substitute the value of $t^{*}$ into the last term to see if neglecting it was justified. Following this procedure we can approximate the mean interval as $\mathrm{t}^{*}$ (see Smith and Smith, 1984, for conditions of validity).

$$
\mathrm{t}^{*}=\mathrm{T}_{\mathrm{R}}-r\left\{\ln \left(1-\frac{\mathrm{S}}{\lambda \mathrm{a} \tau}\right)+\ln \left(1-\frac{\kappa}{\tau}\right)\right\}
$$

Note that the last term does not depend on $\lambda$, and corresponds to the effect of adding a relative refractoriness in the model. Hence this contribution should be of the same value for all conditions where the approximation applies.

Let us return to the example above with $\lambda=1000 \mathrm{~s}^{-1}$, the asymptotic level $\lambda \mathrm{a} \tau$ is $18.56 \mathrm{mV}$ $>\mathrm{S}=12 \mathrm{mV}$ and termwise eqn $(5.3)$ becomes $\mathrm{t}^{*}=1.5+6.03+1.10=7.53+1.10=8.63 \mathrm{~ms}$. The predictions for curves $\alpha$ and $\beta$ at $\lambda=1000 \mathrm{~s}^{-1}$ are $7.53 \mathrm{~ms}, 8.63 \mathrm{~ms}$ resp., compared with the simulated values of $7.25,8.32$ resp. So the method produces a slight overestimate. The approximation does not work as well for $\lambda=800 \mathrm{~s}^{-1}$. Here, $\mathrm{t}^{*}=12.1$ compared with the simulated value of 10.5 for curve $\beta$. The reason is that the asymptotic level is $14.8 \mathrm{mV}$ and is not sufficiently above the threshold $\mathrm{S}=12 \mathrm{mV}$.

The lengthening of the mean interval from curve $\beta$ to curve $\gamma$ can be approximated in a simple way uncler certain conditions. In the simulations with $\lambda=800 \mathrm{~s}^{-1}$, for curve $\beta$ the mean interval (standard deviation) were $10.50(4.94) \mathrm{ms}$, while for curve $\gamma$ they were $11.92(5.24) \mathrm{ms}$. Due to rule 3 . of the model, the AHP is shut off once the trajectory crosses the resting level. When the AHP is terminated by the FIRST arriving EPSP, the first occurrence time of the EPSP's approximates the effect of an $\Lambda I I P$ on the ISI's. Adding this exponential random variable to the ISI's for curve $\beta$ gives for curve $\gamma$ an approximated mean (standard deviation) of $11.75(5.10)$, a slight underestimate.

As it was pointed above, it is a difficult task to find an analytical solution for the FPT problem in this model. Ilowever, the solution is available for some choice of parameters. Fo example, in 
he case consiclered in the second set of simulations, when the size of the EPSP was bigger than the distance between the resting potential and the threshold. 'Two jumps are needed to cross the threshold in the first phase after the previous action potential. This phase, $T_{B}$, last till the trajectory of the AHP becomes close to the resting level, $S-x(t)<a(t)=a(1-\exp (-t / \kappa))$. Under this condition the probability density function of ISI's takes the form

$$
\begin{array}{ll}
f(t)=\lambda^{2} e^{-\lambda t} t & \text { for } t \in\left[0, T_{B}\right] \\
f(t)=\lambda e^{-\lambda t} & \text { for } t>T_{B}
\end{array}
$$

For $\lambda=50 \mathrm{~s}^{-1}$ and $\mathrm{T}_{\mathrm{B}}=\mathrm{T}_{\mathrm{E}}=100$ the distribution (5.4) practically coincides with an Erlang density $(k=2)$ as is illustrated on Fig. 6. The difference between the simulated and the approximated histograms is so small that cannot be documented in this figure. This also testifies the correctness of the simulation procedure. Using (5.4) the moments of ' $\mathrm{I}^{*}$ can be computed,

$$
\begin{gathered}
\mathrm{E}\left(\mathrm{T}^{*}\right)=\frac{2-\exp \left(-\lambda \mathrm{T}_{\mathrm{B}}\right)}{\lambda} \\
\operatorname{Var}\left(\mathrm{T}^{*}\right)=\frac{2+\lambda \exp \left(-\lambda \mathrm{T}_{\mathrm{B}}\right)\left[\mathrm{T}_{\mathrm{B}}^{3}\left(\lambda^{2}-1\right)-2 \mathrm{~T}_{\mathrm{B}}\right]-\exp \left(-2 \lambda \mathrm{T}_{\mathrm{B}}\right)}{\lambda^{2}} \\
\operatorname{CV}\left(\mathrm{T}^{*}\right)=\frac{2-\exp \left(-2 \lambda \mathrm{T}_{\mathrm{B}}\right)+\lambda \exp \left(-\lambda \mathrm{T}_{\mathrm{B}}\right)\left[\mathrm{T}_{\mathrm{B}}^{3}\left(\lambda^{2}-1\right)-2 \mathrm{~T}_{\mathrm{B}}\right]}{4-4 \exp \left(-\lambda \mathrm{T}_{\mathrm{B}}\right)+\exp \left(-2 \lambda \mathrm{T}_{\mathrm{B}}\right)}
\end{gathered}
$$

The generalization on the case in which $k_{1}$ EPSP's is needed for the crossing of the threshold during ' $T_{B}$ period and after its end only $k_{2}$ EPSP's is the minimal number $\left(k_{2}<k_{1}\right)$ would be straight.forward.

The output frequencies computed by using the approximation (5.5) were compared with those which were obtained in the second set of simulalions. The differences were negligible, with a tendency 
to underestimate by the approximation (5.5) for lower input frequencies $\left(\lambda=2.5 \mathrm{~s}^{-1}\right.$; the simulated frequency and approximated frequency were $2.139 \mathrm{~s}^{-1}$ and $2.041 \mathrm{~s}^{-1}$, respectively) and to overestimate for higher input frequencies $\left(\lambda=500 \mathrm{~s}^{-1} ; 174.0 \mathrm{~s}^{-1}\right.$ and $181.8 \mathrm{~s}^{-1}$, simulated frequency and approximated frequency, respectively).

\section{Discussion}

The parameters of the AHP show considerable variation among different types of neurons. For example, in the experiments of Weems and Szurszewski (1978) on neurons of the inferior mesenteric ganglion (ING) of guinea pigs the amplitude of the $\Lambda$ HP ranged from 8 to $20 \mathrm{mV}$ and the average of its total duration was $175 \mathrm{~ms}$; while Grantyn and Grantyn (1978) observed an average amplitude of the AIIP of only $1.8 \mathrm{mV}$ and with an average total duration of $47 \mathrm{~ms}$ in cat ocular motoneurons. The model of $\Lambda \mathrm{HP}$ in this article chose a functional form similar to a Gamma probability density function. This assumption appears reasonable in that many experimental records of AHP resemble an inverted Gamma function (e.g., Schmidt, 1984, Ganong, 1985). There are however some exceptions. Grantyn and Grantyn (1978) presented a few experimental records where the course of the AHP is humped and would not seem to be well approximated by the shape of a Gamma function. Such a discrepancy may be connected with some other property, e.g. synaptic activity or delayed depolarization, rather than being a property of the AHP process itself.

Another approach to describing the AHP is through the use of timevarying conductances in the circuit morlel for the neuron. This approach has a long history (for references see Jack et al., 1975). A more recent stochastic example (Smith and Goldberg, 1986) used a modification of Kernell's model (Kernell, 1968) with cumulative AHP. This model reproduced many features of the steady state discharge of peripheral vestibular afferents, e.g. ISI statistics during natural and electrical stimulation, and the increase in interspike interval following an interposed"shock. The cumulative AHP aspect of this model makes the resultant spike train a non-renewal process.

In a followup study, Smith and Chen (1986) examined this model by simulations with and 
withont cumulative effects. The cumulative effect was due to the summing of the residual activity of the potassium conductance of the preceding interval. The statistical properties of the ISI's that are independent of the serial ordering of the ISI's, e.g. mean, CV, show only slight difference between the two cases. Several of their results, e.g. firing frequency vs $\lambda$, are similar to the results presented here, as well as to some deterministic models with $\Lambda H P$ (MacGregor and Oliver, 1974). A difference with the present. study is found with order dependent statistical measures. Here we find no significant first order serial correlations for the simulations examined, Smith and Chen (1986) found that a significant negative serial dependency results from cumulative AHP at high to moderate discharge rates. The reason for the difference in these results is due to the method of modeling the $\Lambda H P$ and its dependence on the previous trajectory. The amplitude II in this study depends on the previous trajectory only via its final value $\mathrm{X}_{\mathrm{F}}$. The final value and length of that ISI are not strongly related, hence the length of the preceding interval has no systematic affect on the length of the following one.

The previous final level of the trajectory of the membrane potential following the spike was also used in the neuronal model of Sugano (1984). The author simplified the situation in such a way that only the value of the initial depolarization depended on the preceding final level, as compared with setting the maximum AHP in this study. In our model of AHP the amplitude is a random variable since the preceding final level is a random variable. A histogram of AHP amplitude $\mathrm{H}$ obtained via computer simulation is shown in Fig. 4a. We can compare it with the histogram of O'Neill et al., (1986) who experimentally measured the minimum of the action potential wave in very slow discharging neurons. The shapes of both histograms are rather similar and also their standard deviations do not differ substantially $(0.227$ respectively $0.225 \mathrm{~ms})$.

One assumption in this paper is the linear relationship between the final value of the membrane potential, $\mathrm{X}_{\mathrm{F}}$, and the magnitude of the AHP. This relationship was determined primarily for different steady state values of $\mathrm{X}_{\mathrm{F}}$ due to current injection and may not be as applicable for the synaptically driven neuron. Some support for this assumption comes from Fig. 3 of Weems and Szurszewski (1978), where the AIIP voltage trajectories due to repetitive stimulation by injected 
currents and due to synaptic activation were quite similar. In any case, the linear relationship for injected currents has been found in several types of neurons (e.g. Coombs et al., 1955, Kuno, 1959, Weems and Szurszewski, 1978). It follows from the linear transformation between the final depolarization $X_{F}$ and the amplitude $H$ that if the theoretical distribution of $X_{F}$ is available then that of II can be computed. Tsurui and Osaki (1976) derived an integral equation for the final value $\mathrm{X}_{\mathrm{F}}$ in the model (3.1). However, the introduced modifications would hardly permit to find a theoretical counterpari to the results presented in Fig. 4a. However, for all the simulations in which the size of EPSP was only a small fraction of the distance between the resting level and the threshold potential, the hisiograms of $\mathrm{H}$ were monotonous with the highest number of cases in the first bin.

'Tuckwell (1978) introduced an AHP into Stein's model for excitation only (3.1) by using an exponentially decaying threshold. In his morlel no variability in AHP nor dependency on previous firing was present. We compared Tuckwell's results with those obtained for our method of introducing an AIIP. For model (3.3), the frequency transfer characteristic is illustrated in Fig. 3. Although the approaches are quite different, the conclusion is practically identical: AHP has not much effect on the overall shape of the frequency transfer characteristic and on the moments of the ISI's in general. A similar lack of effect on ISI statistics was seen in Stein's model with a reversal potential and a random initial depolarization below the resting level (Lánský and Musila, 1991). The main effects are seen at high firing rates and in the removal of short intervals in the ISI histogram.

An example of the influence of the AHP on the shape of ISI distribution is shown in Fig. 6. It also supports the remarks of Eccles et al. (1958) that in some neurons a strong $\Lambda H P$ may substantially retard the neuronal firing. In the present model, the effects of the AHP increase with increasing EPSP size as it follows from the comparison of both sets of simulations. Confirmation of this result with the other methods of describing an AHP would help determine its physiological validity.

ACKNOWLWICGEMENT: Supported in part by Office of Naval Research Grant N00014-90-J-1646-1, NAS-CSAS scientist exchange program and Czechoslovak Academy of Sciences grant \#71143. 
References

Brock LG, Coombs JS, Eccles JC (1952) The recording of potentials from motoneurones with an intracellular electrode. J Physiol 117:431-460

Coombs JS, Eccles JC, Fat P (1955) The electrical properties of the motoneurone membrane. J Physiol 130:291-325

Correia M.J, Landolt JP (1977) A point process analysis of the spontaneous activity of anterior semicircular canal units in the anesthetized pigeon. Biol Cybern 27:199-213

Clay JR, Goel NS (1973) Diffusion models for firing of a neuron with varying threshold. J Theor Biol 39:633-644

Coleman R., Gastwirth JL (1970) Some models for interaction of renewal processes related to neuron firing. J $\Lambda$ ppl Prob 6:38-58

Ecrles JC: (1957) The physiology of nerve cell. Johns Ilopkins Press, Baltimore

Eccles JC, Eccles RM, Lundberg A (1958) The action potentials of the alpha motoneurones supplying fast and slow muscles. J Physiol 142:275-291

Floyd K, llick VE, Holden AV, Koley J, Morrison JFB (1982) Non-Markov negative correlation betwcen interspike intervals in mammalian efferent discharge. Biol Cybern 45:89-93

Ganong WP (1985) Review of medical physiology, 12th edition. Lange Medical Publications, Los Altos Geisler CD, Goldberg JM (1966) A stochastic model for the repetitive activity of neurons. Biophys J 6:5:5-69

Grantyn R. Grantyn A (1978) Morphological and electrophysiological properties of cat abducens motoneurons. Exp Brain Res 31:249-274

Jack JJB, Nobel B, Tsien RW (1975) Electric current flow in excitable cells. Oxford Univ Press, Claredon

Kataynma $Y(1971)$ Recovery course of excitability in a single neuron of Onchidium Verruculatum. J Pxper Biol 54:471-484 
Kernell D (1968) The repetitive impulse discharge of a simple neuron model compared to that of spinal moloneurones. Brain Res 11:685-687

Kuno MI (1959) Excitability following antidromic activation in spinal motoneurones supplying red muscles. J Physiol 149:374-393

Lev-'Iov A, Miller JP, Burke RE, Rall W (1983) Factors that control amplitude of EPSPs in dendritic Ireurons. J Neurophysiol 50:399-412

Levine NW (1982) Retinal processing of intrinsic and extrinsic noise. J Neurophysiol 48:992-1010

LAánský P (1981) On approximations of Stein's neuronal model. J Theor Biol 107:631-647

Lánský P, Musila M (1991) Variable initial depolarization in Stein's neuronal model with synaptic reversal potentials. Biol Cybern 64:285-291

Lánsky P, Radil T (1987) Statistical inference on spontaneous neuronal discharge patterns. Biol Cybern 55:299-311

Lánsky P. Radil-Weiss T, IIrubant K, Indra M (1981) $\Lambda$ note to the selective interaction models of single nemron firing trains In: Barlfai $\mathrm{P}$, Tomko $\mathrm{J}$ (Eds.) Point processes and queuing problems. North-Ilolland, Amsterdam

Lansky P, Smith CE (1989) The effect of a random initial condition in neural first-passage-time models. Math Biosci 93:191-215

Maclonald RL, Pum RYK, Neale EA, Nelson PG (1983) Synaptic interactions between mammalian central neurons in cell culture. I. Reversal potential for excitatory postsynaptic potentials. J Neurophysiol 49:1428-1441

MacGregor R.J, Oliver RM (1974) A model for repetitive firing in neurons. Kybernetik 16:53-64

Musila M, Iánský P (1991) Generalized Stein's model for anatomically complex neurons. BioSystems $25: 179-191$

Neale F $\Lambda$, Nelson PG, MacDonald RL, Christian CN, Bowers LM (1983) Synaptic interactions between mammalian central neurous in cell culture. III. Morphophysiological correlates of quantal synaptic transmission. J Neurophysiol 49:1459-1468 
O'Neill WD, Lin IC, Ma Y-C (1986) Estimation and verification of a stochastic neuron model. IEEE Trans Biomed Engn 33:654-666

Nelson PG, Marshall KC, Pun RYK, Christian CN, Sheriff Jr. WH, MacDonald RL, Neale EA, (1983) Syrraptic interactions between mammalian central neurons in cell culture. II. Quantal analysis of EPSPs. J Neurophysiol 49:1442-1458

Ruch TC, Patton IID, Woodbury JW, Towe AL (1968) Neurophysiology, 2nd edition. Saunders, Iondon

Schmidt RF (ed) (1984) Fundamentals of neurophysiology, 2nd edition. Springer, Berlin

Smith CE, Chen C (1986) Serial dependency in neural point processes due to cumulative afterhyperpolarization, Inst Stat Mimeo Series No. 1691. North Carolina State University, Raleigh

Smith CE, Goldberg JM (1986) $\Lambda$ stochastic afterhyperpolarization model of repetitive activity in vestibular afferents. Biol Cybern 54:41-45

Smith DR., Smith GK (1965) A statistical analysis of the continual activity of single cortical neurones in the cat unanaestatized isolated forebrain. Biophys J 5:47-74

Smith CE, Smith MV (1984) Moments of voltage trajectories for Stein's model with synaptic reversal potentials. J Theor Neurobiol 3:67-77

Stein RB (1965) A theoretical analysis of neuronal variability. Biopliys J 5:173-195

Sugano N (1984) Effect of doublet impulse sequences on the transient and steady responses in the computer simulated nerve cell. Biol Cybern 51:123-128

Surmeier D.J, IIonda CN, Willis WD (1989) Patterns of spontaneous discharge in Primate Spinothalamic Neurons. J Neurophysiol 61:106115

Tipans I, Lavenclels E (1983) A mathematical model of afterdepolarization. Gen Physiol Biophys 2:163167

Tsurui A, Osaki S (1976) On a first-passage time problem for a cumulative process with exponential decay. Stoch Proc $A$ ppl 4:79-88 
Tuckwell IIC (1976a) Firing rates of motoneurones with strong synaptic excitation. Biol Cybern 24:147-152

Tuckwell HC (1976b) On the first-exit time problem for temporally homogeneous markov processes. J Appl Prob 13:39-48

'Tuckwell IIC (1978) Reccurent inhibition and afterhyperpolarization: Effects on neuronal discharge. Biol Cybern 30:115-123

'Tuckwell II(: (1979) Synaptic transmission in a model for stochastic neural activity. J Theor Biol 77: 65-81

Tuckwell HC: (1988) Introduction to theoretical neurobiology, Vol.2: Nonlinear and stochastic theories. Cambridge Univ. Press, Cambridge

'Tuckwell HC (1989) Stochastic processes in the neurosciences. SIAM, Philadelphia

Tuckwell IIC, Wan FYM (1984) First-passage times of Markov processes to moving barriers. J Appl Prob 21:695-709

Vasudevan R, Vittal PR (1982) Time-dependent barriers and first-passage times - Different types of neuronal models. Neurol Res 4:63-87

Walloe $\mathrm{I},(1970)$ On the transmission of information through sensory neurons. Biophys $\mathrm{J}$ 10:745-763

Wecms WA, Szurszewski JH (1978) An intracellular analysis of some intrinsic factors controlling neural output from inferior mesenteric ganglion of guinea pigs. J Neurophysiol 41:305-321

Wilbur A.l, Rinzel J (1982) An analysis of Stein's model for stochastic neuronal excitation. Biol Cybern $45: 10 \overline{7}-115$

Willur AJ, Rinzel J (1983) A theoretical basis for large coefficient of variation and bimodality in neuronal interspike interval distribution. $\mathrm{J}$ Theor Biol 105:345-368

Yamamoto M, Nakahama II (1983) Stochastic properties of spontaneous unit discharges in somatosensory cortex and mesencephalic reticular formation during sleep-waking states. J Neurophysiol 19:1182-1198 


\section{'Text to figures}

Fig. 1. A schematic presentation of the action potential and the following afterhyperpolarization.

Fig. 2. A schematic sample trajectory of the neuronal model given by equation (3.3)

Fig. 3a. Dependency of the mean output firing rate on the input frequency $\lambda$. Case $\alpha$ corresponds to the model (3.1), $\beta$ stands for the model (3.3) and $\gamma$ for the model (3.3) with introduced AHP model. For the other parameters see text.

Fig. 3b. Dependency of the coefficient of variation CV of the ISI's on the output frequency $f$. The cases $\alpha, \beta$ and $\gamma$ as in Fig. 3a. For the other parameters see text.

Fig. 3c. Pearson plot of coefficient of skewness $\left(\beta_{1}\right)$ versus coefficient of kurtosis $\left(\beta_{2}\right)$ of the ISIs in dependency on the input frequencies $\lambda=200,400,600,800$ and $1000 \mathrm{~s}^{-1}$. Different symbols indicate the type of the model, o - Stein's model (3.1), $\mathrm{x}$ - model (3.3), $\Delta$ - model (3.3) with introduced AIIP.

Fig. 1a. Dependency of the statistical characteristics of the minimal level of the membrane potential $\mathrm{X}_{\mathrm{M}}$ on the input frequency $\lambda$. Means denoted by crosses, standard deviations denoted by circles. For the other parameters see text.

Fig. 4b. Histogram of the AHP amplitudes $\mathrm{H}$ as computed from the equation (2.4) for the input frequency $\lambda=200 \mathrm{~s}^{-1}$. For the other parameters see text.

Fig. Ac. Histograms of the AHP maximal amplitudes $X_{M}$ for the input frequency $\lambda=200 \mathrm{~s}^{-1}$ and $\lambda=$ $1000 \mathrm{~s}^{-1}$. For the other parameters see text.

Fig. 5. Percentage difference of output frequencies between the model (3.3), $\mathrm{f}_{1}$, and the model with AIIP, $f_{2}$, plotted against the input frequency $\lambda$. The difference is computed as $100\left(f_{1}-f_{2}\right) / f_{1}$. For the other parameters see text.

Fig. 6. The histograms of the ISI. The model (3.1), the exponential density denoted by triangles. The simulated model (3.3) denoted by circles. The model (3.3) with AIP denoted by crosses, the simulated results and the approximation computed in accordance with (5.4) are undistinguishable. 


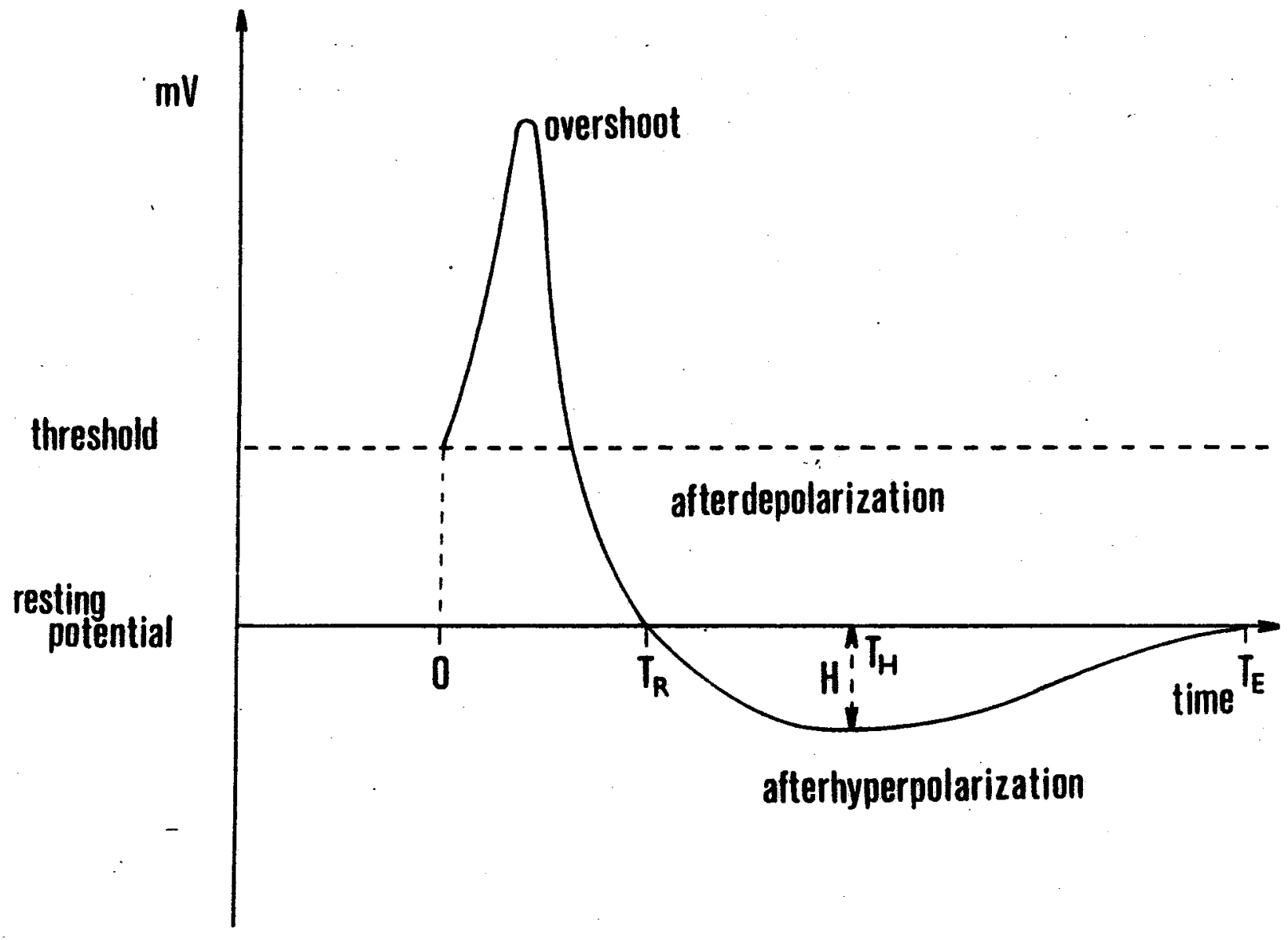

Fig. 1

VOLTAGE

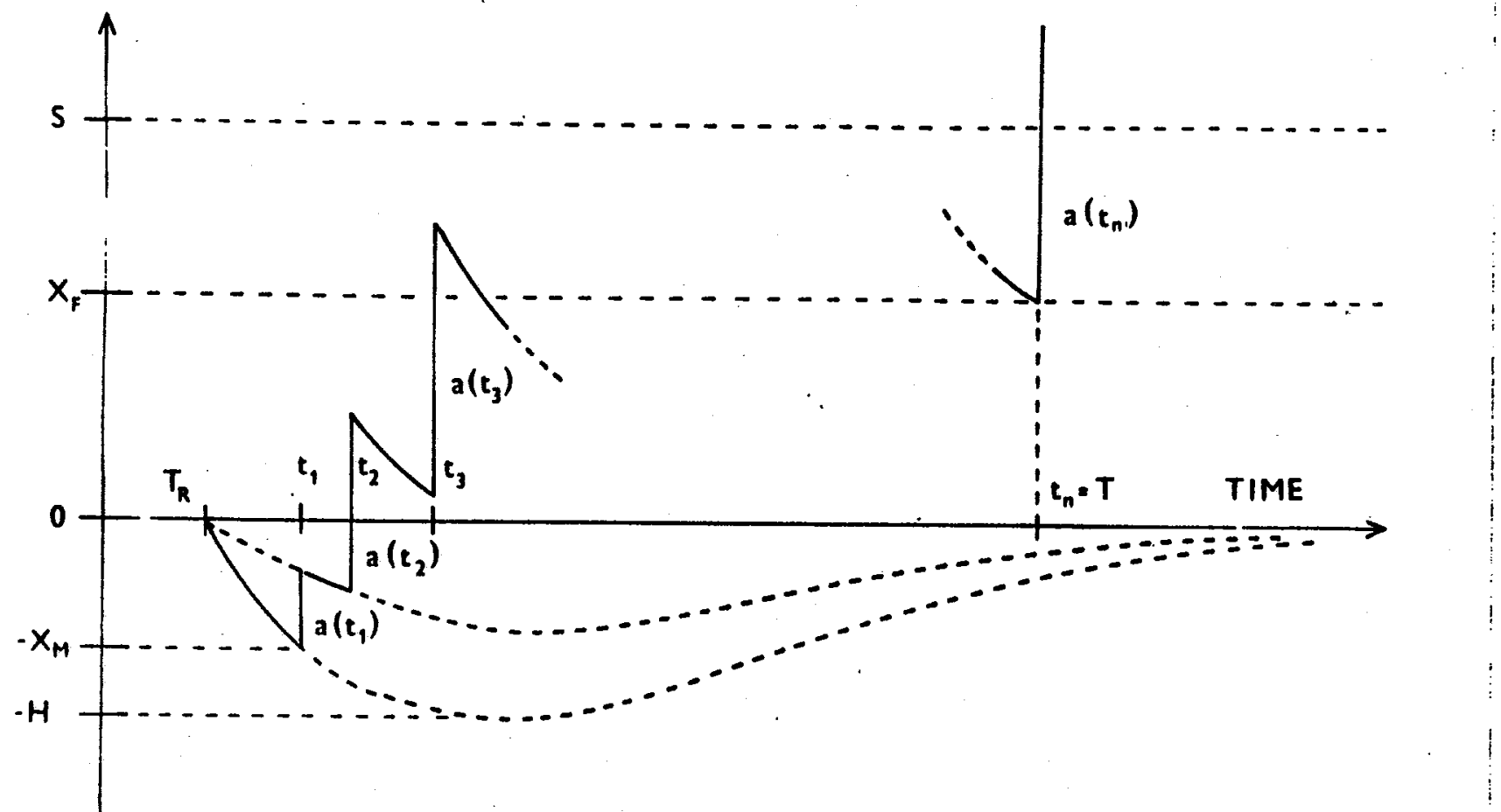

Fig. 2 

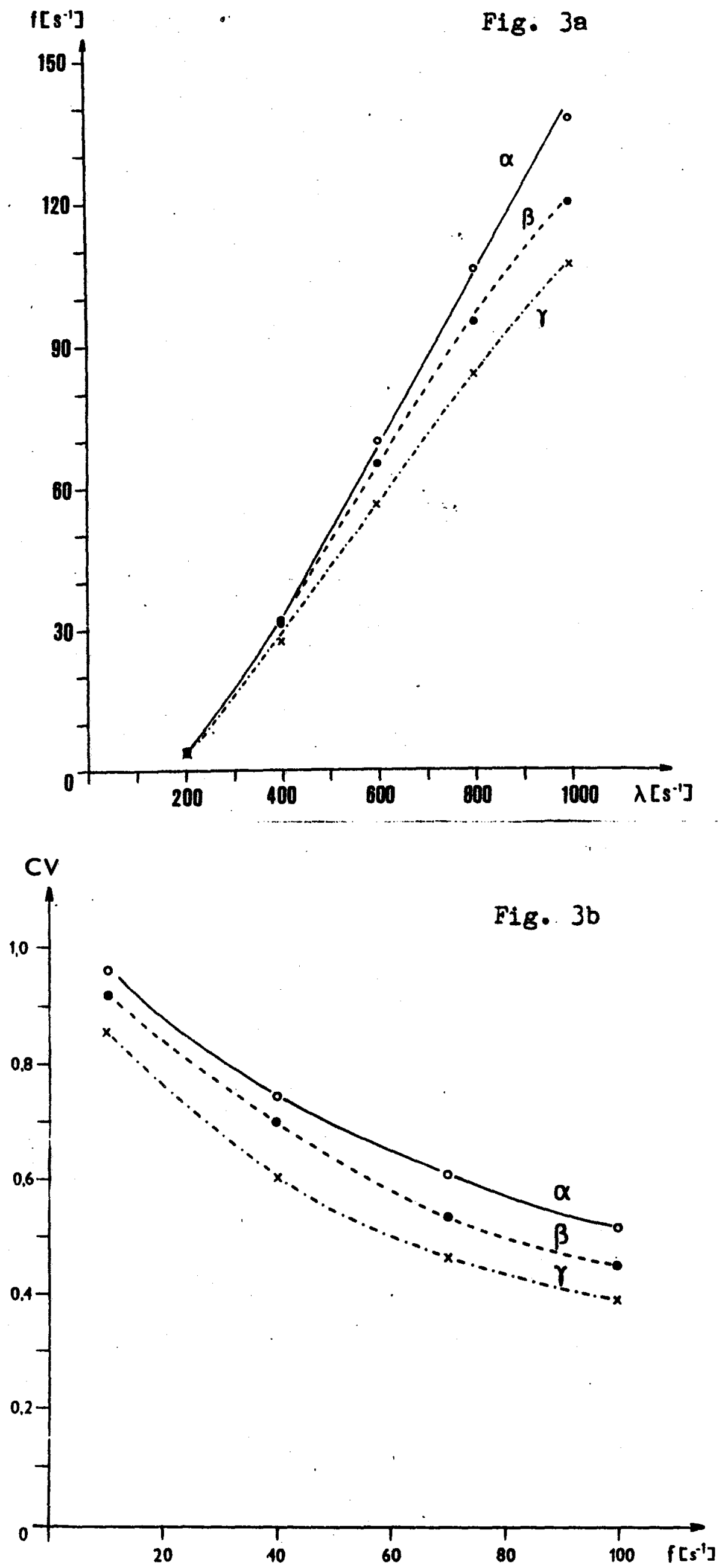

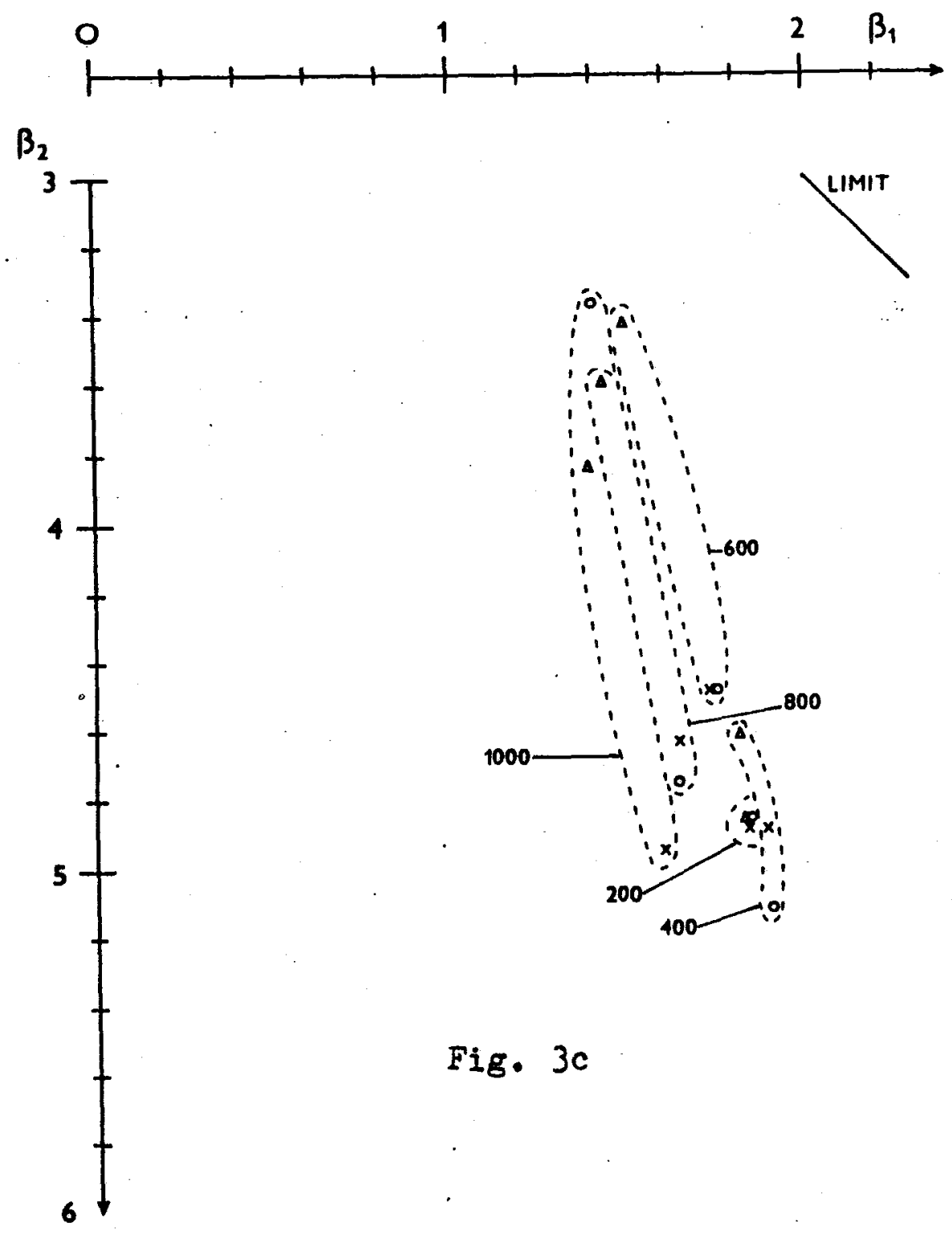

Fig. $3 c$

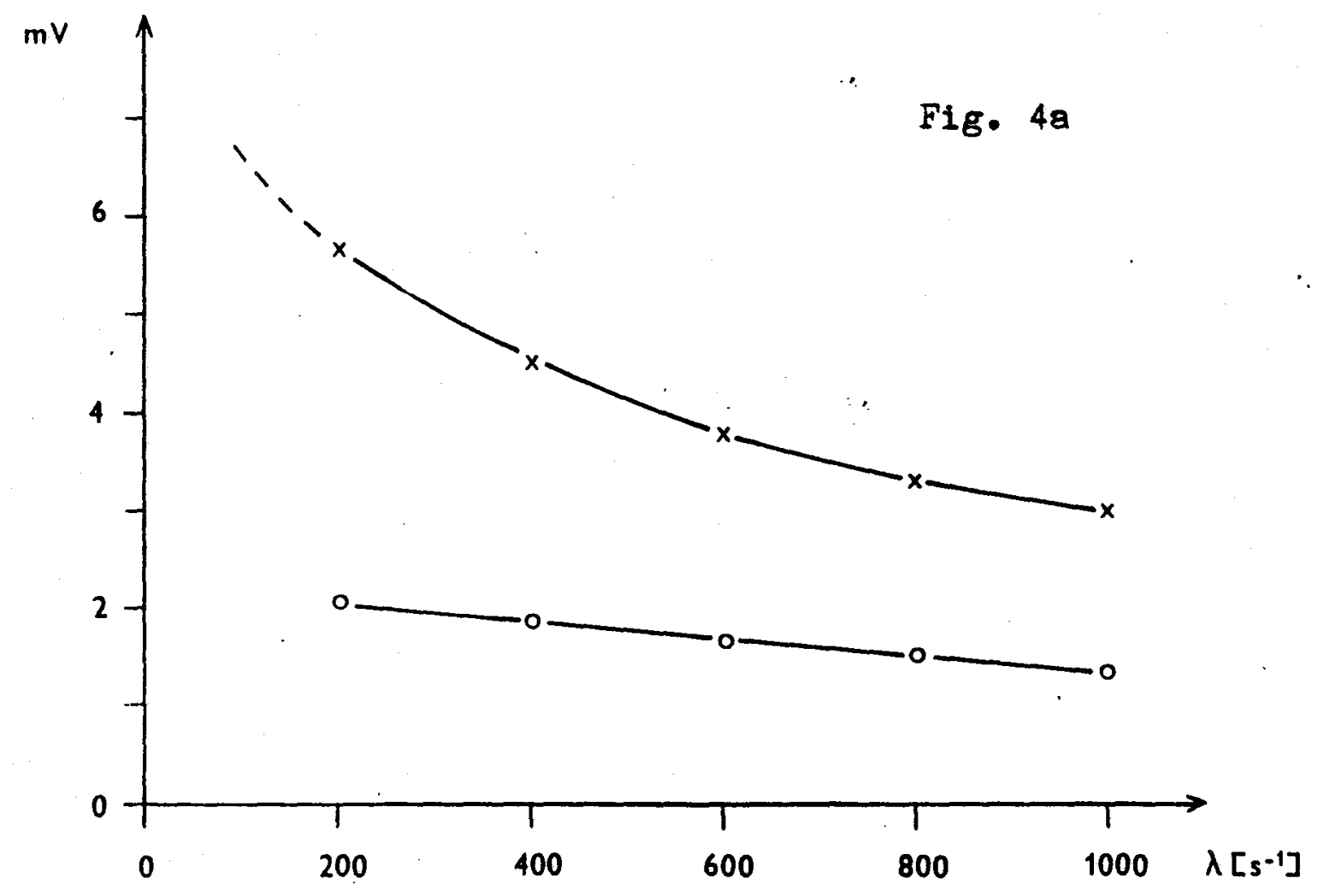




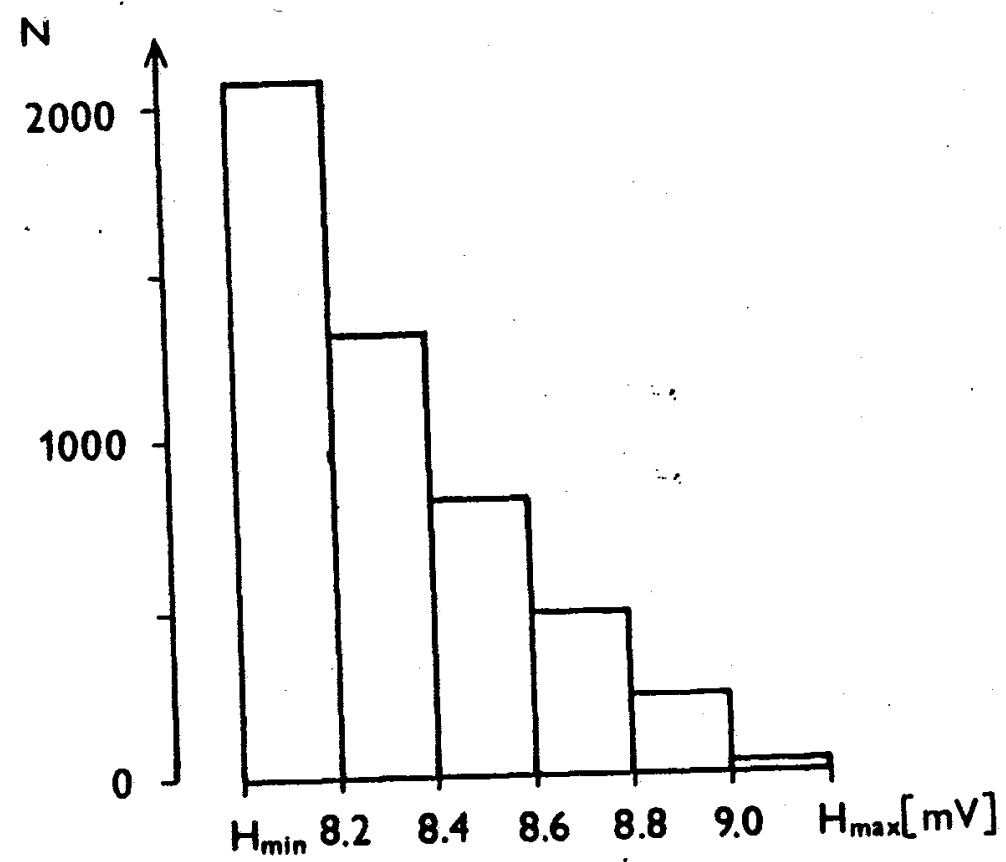

Fig. 4b

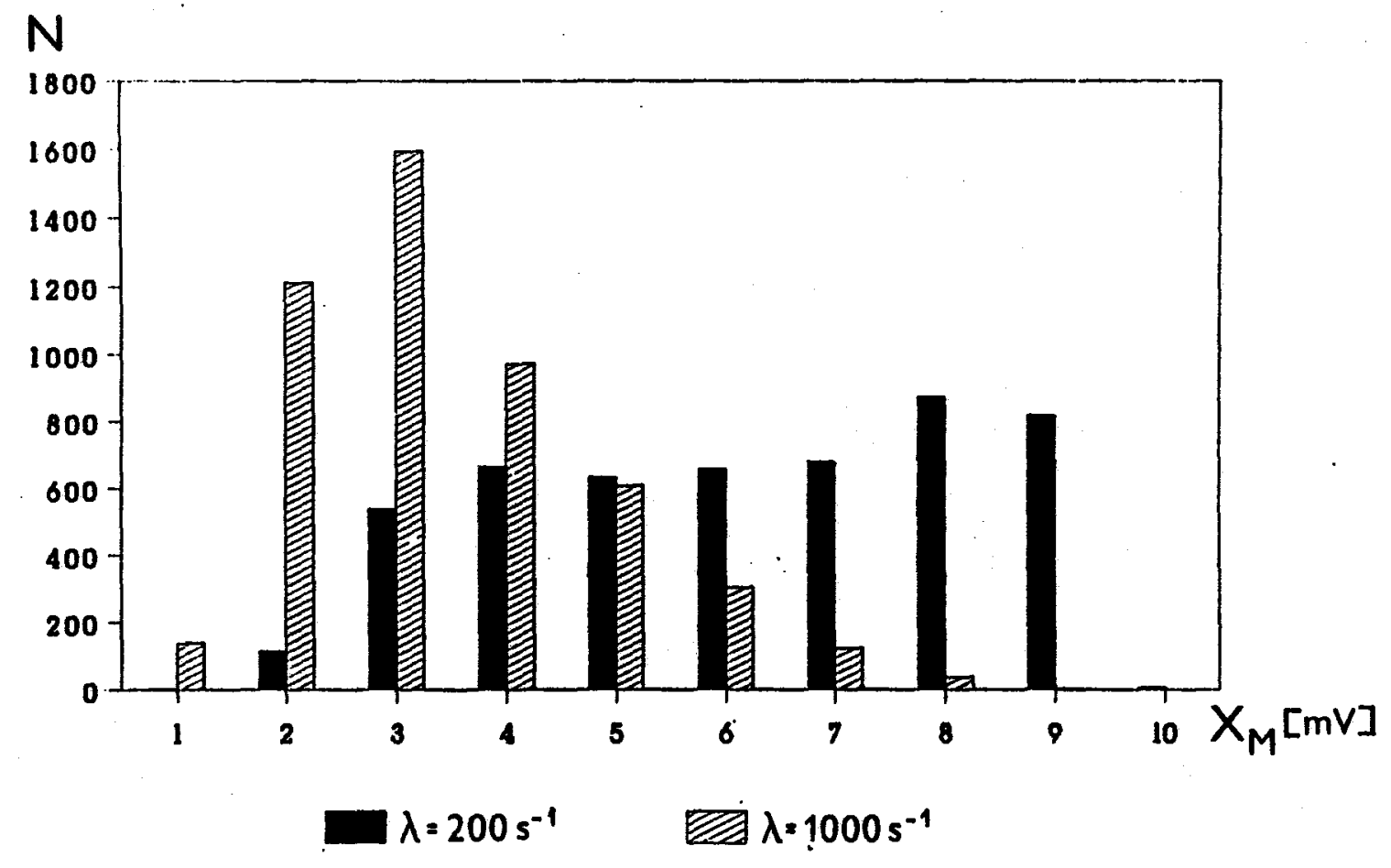

Fig. 4c 


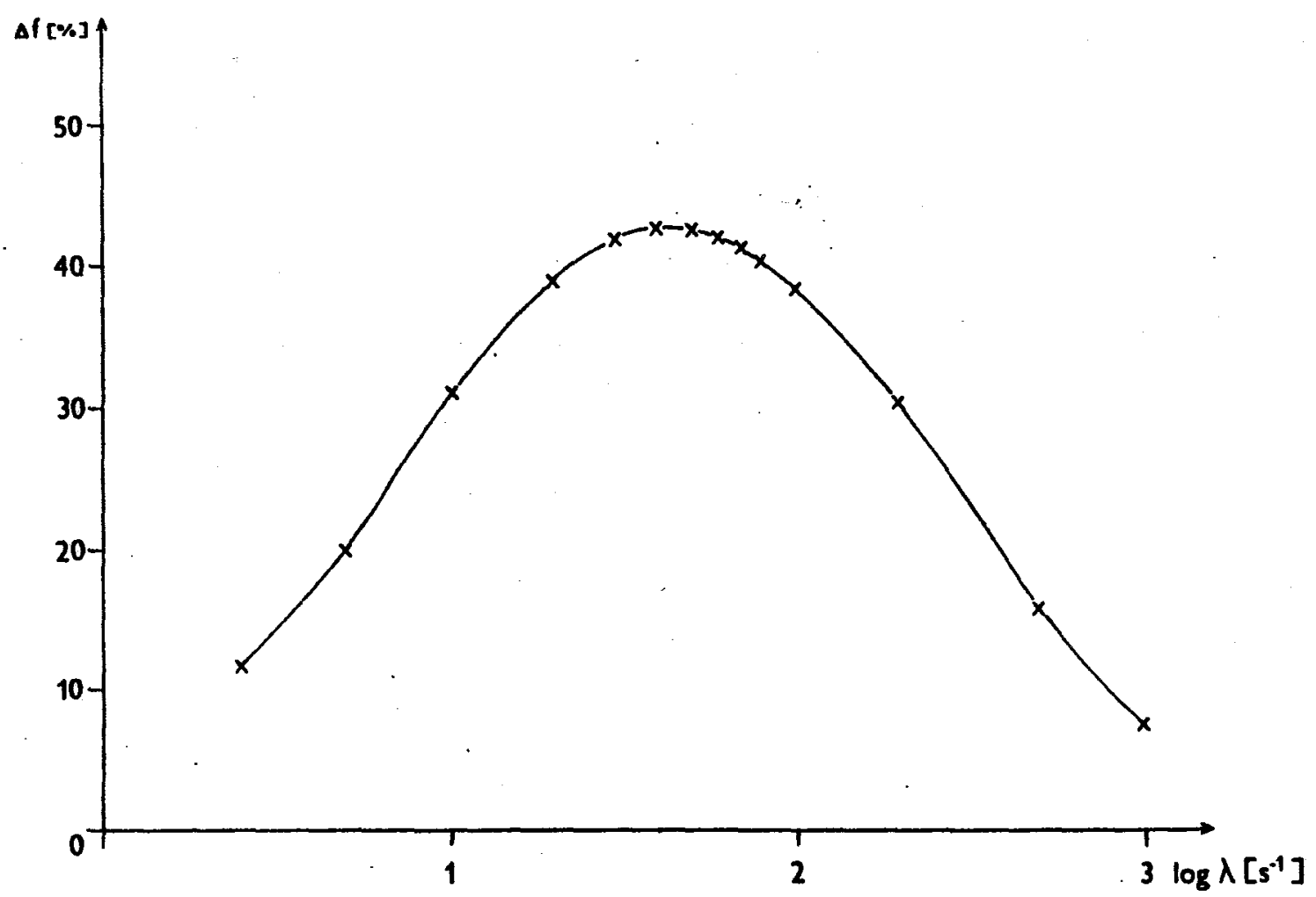

Fig. 5

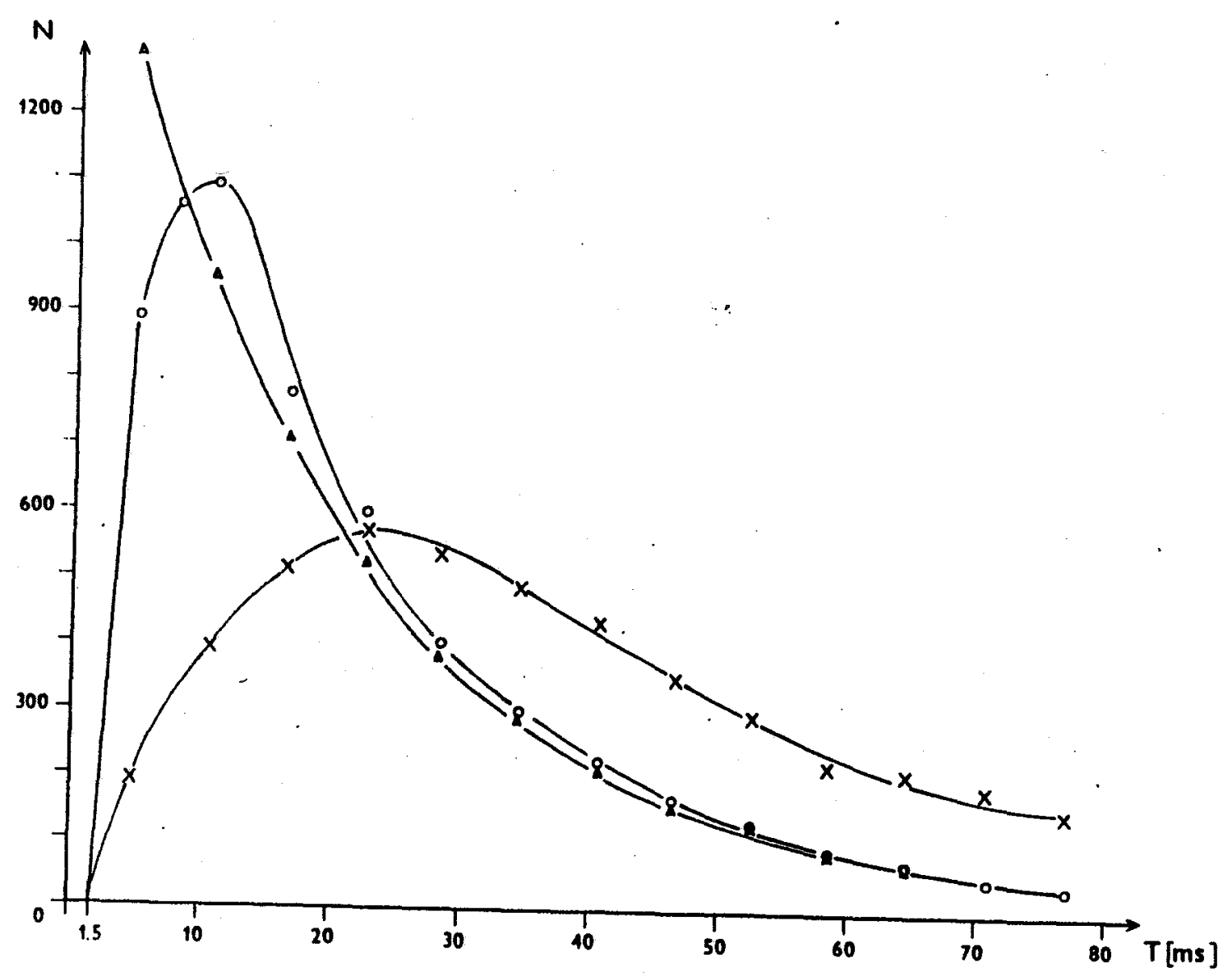

Fig. 6 